\title{
Crustáceos decápodes de água doce com ocorrência no Vale do Ribeira de Iguape e rios costeiros adjacentes, São Paulo, Brasil
}

\author{
Sérgio Schwarz da Rocha \& Sérgio Luiz de Siqueira Bueno
}

Departamento de Zoologia, Instituto de Biociências, Universidade de São Paulo. Rua do Matão, Travessa 14, 101, Cidade Universitária, 05508-900 São Paulo, São Paulo, Brasil.E-mail: ssrocha@superig.com.br; sbueno@ib.usp.br

\begin{abstract}
Freshwater decapod crustaceans from Ribeira de Iguape river basin and adjacent coastal areas, São Paulo Brazil. This paper contributes to the knowledge of the species richness and distribution of decapod crustaceans at the Ribeira de Iguape river basin, south of São Paulo State, Brazil. Seven protected areas (E.E. Juréia-Itatins, P.E. Ilha do Cardoso, P.E. Jacupiranga, P.E. Campina do Encantado, P.E. Carlos Botelho, P.E. Intervales and PETAR) were investigated, covering most of the Mata Atlântica remaining in the Ribeira de Iguape basin. Samples were taken during each season of the years 2000 and 2001 using two sampling methods (sieves and traps). Six species of palaemonid shrimps, three atyid shrimps, five aeglid anomuran crabs, and three trichodactylid and one grapsid crabs were collected.
\end{abstract}

KEY WORDS. Distribution, field survey, richness, taxonomy.

RESUMO. Este trabalho contribui para o conhecimento da riqueza de espécies de crustáceos decápodes do vale do Ribeira de Iguape, região sul do estado de São Paulo. Foram realizadas amostragens em seis Parques Estaduais (Campina do Encantado, Intervales, PETAR, Carlos Botelho, Jacupiranga e Ilha do Cardoso) e na Estação Ecológica Juréia-Itatins, abrangendo grande parte do remanescente de Mata Atlântica da região da bacia do rio Ribeira de Iguape. As coletas foram realizadas sazonalmente durante os anos 2000 e 2001, empregando dois métodos de amostragem (peneira e armadilha com isca). Foram registradas 18 espécies de crustáceos decápodes: Palaemonidae (6), Atyidae (3), Aeglidae (5), Trichodactylidae (3) e Grapsidae (1).

PALAVRAS CHAVE. Distribuição, levantamento, riqueza, taxonomia.

Há um número considerável de trabalhos publicados sobre a fauna de crustáceos bentônicos brasileiros nos últimos vinte anos, a maioria, porém, diz respeito a espécies marinhas e estuarinas. Os crustáceos de água doce têm recebido pouca atenção da comunidade científica brasileira. Alguns dos estudos mais significativos e pioneiros sobre crustáceos decápodes foram produzidos no final do século XIX (MüLler 1880, 1892a,b, Ortmann 1897, von Ihering 1897) e início do século XX (Luederwaldt 1919a,b, Schmitt 1942, Oliveira 1945, SaWaya 1946). Desde então, a escassez de trabalhos de sistemática e inventários faunísticos, bem como de dados biológicos, têm contribuído para que continuássemos, por muito tempo, a ter um conhecimento fragmentário sobre os grupos de decápodes existentes nos principais corpos de água do Brasil.

Num passado mais recente, merecem destaque as revisões taxonômicas de camarões palemonídeos dulciaqüícolas, anomuros eglídeos e caranguejos tricodactilídeos, feitas por Holthuis (1954), Bond-Buckup \& Buckup (1994) e Magalhães \& TÜRKAY (1996), respectivamente. Recentemente, os decápodes dulcícolas brasileiros foram tema de um manual editado por MeLo (2003).
No estado de São Paulo, entretanto, o atual conhecimento dos decápodes de ambientes dulcícolas é ainda insuficiente. A síntese mais recente foi publicada por MagalHães (1999b), que registrou 32 espécies nas sub-bacias hidrográficas desse estado. Outros estudos abordaram os camarões atiídeos (GALVÃo \& Bueno, 2000), anomuros eglídeos (BOND-Buckup \& BuCKup, 1994) e a redescrição de novas espécies de camarões palemonídeos (Melo et al., 1988). Aspectos da fisiologia (Moreira et al., 1980; Elmor et al., 1981; McNamara, 1981; McNamara \& Sesso, 1982), biologia reprodutiva e desenvolvimento pós-embrionário (Bueno \& Rodrigues 1995, Melo \& Brossi-Garcia 1999, Mossolin \& Bueno, 2002), crescimento alométrico (Mossolin \& Bueno 2003) e parasitismo (Rocha \& BuENo 2000), também foram estudados para camarões palemonídeos do gênero Macrobrachium Bate, 1868.

Magalhães (1999b) alertou para a necessidade de intensificação dos estudos e do esforço de coleta para melhor caracterização da riqueza de espécies de decápodes do estado de São Paulo, particularmente para a região sul, onde o Vale do Ribeira é uma das áreas prioritárias. Esta região engloba a bacia do rio Ribeira de Iguape, representando uma região ecologicamente

Revista Brasileira de Zoologia 21 (4): 1001-1010, dezembro 2004 
relevante por preservar uma importante área remanescente da Mata Atlântica. Essa região, entretanto, encontra-se sob contínuo processo de degradação em virtude do acelerado crescimento urbano, ocupação antrópica para a agricultura, ou ainda estabelecimento de atividade industrial. Como conseqüência, os habitats naturais podem ficar seriamente comprometidos ou mesmo deixarem de existir. Como resultado desse processo, populações inteiras de diversas entidades biológicas, inclusive de crustáceos decápodes, podem desaparecer completamente.

O presente estudo fornece informações sobre a riqueza e distribuição das espécies de decápodes em sete áreas de conservação ambiental no Vale do Ribeira de Iguape. Os dados aqui apresentados contribuem para o incremento do conhecimento da fauna de crustáceos do estado de São Paulo, podendo servir de ferramenta ao monitoramento e manejo futuro das espécies registradas na região.

\section{MATERIAL E MÉTODOS}

Sete áreas de proteção ambiental foram estudadas sazonalmente, durante os anos de 2000 e 2001, abrangendo grande parte do remanescente de Mata Atlântica da região da bacia do rio Ribeira de Iguape e áreas costeiras adjacentes. São elas, a Estação Ecológica Juréia-Itatins e os Parques Estaduais Campina do Encantado, Intervales, Turístico do Alto Ribeira (PETAR), Carlos Botelho, Jacupiranga e Ilha do Cardoso (Figs 1 e 2). Foram amostrados quarenta e três pontos, nove deles situados no interior de cavernas do PETAR, Intervales e Jacupiranga.

Foram utilizados dois métodos de captura. Um envolveu o uso de peneiras (malha $2 \mathrm{~mm}$ ) para capturar os indivíduos associados à vegetação marginal ou escondidos no leito do rio; o outro envolveu o uso de armadilhas iscadas com coração de frango e colocadas no leito do rio no final do período da tarde de um dia e recolhidas na manhã do dia seguinte. O emprego dos dois métodos de captura visou otimizar os resultados de coleta no sentido de amostrar indivíduos que ocupam diferentes microhábitats (vegetação marginal e fundo de rio) e/ou que são mais ativos em períodos distintos do dia.

Os animais capturados eram fixados em álcool 70\% nos próprios locais de coleta e acondicionados em frascos plásticos com tampa e etiqueta de identificação. No laboratório, os espécimes foram identificados até o nível de espécie com o auxílio de chaves dicotômicas disponíveis nos trabalhos de HoLthuis (1952) para camarões palemonídeos, Hoвbs \& HaRT (1982) e Villalobos (1959) para camarões atiídeos, BOND-Buckup \& BucKuP (1994) para anomuros eglídeos e Melo (1996) para caranguejos grapsídeos. As identificações dos caranguejos tricodactilídeos foram baseadas no sistema proposto por MAGALHÃEs \& TÜRKAY (1996). Além desses trabalhos, foram consultadas as publicações de Genofre \& Lobão (1978), Kensley \& Walker (1982), Melo et al., (1988) e Ostrovski, et al. (1996), contendo descrições de novas espécies para o estado de São Paulo, não contempladas nas chaves de identificação dos trabalhos mencionados acima.
O material estudado encontra-se depositado no Museu de Zoologia da Universidade de São Paulo, São Paulo, Brasil (MZUSP). Informações da coleção de crustáceos desse museu foram utilizadas na complementação do estudo de distribuição das espécies na região estudada.

\section{RESULTADOS}

A tabela I apresenta a listagem das espécies de decápodes coletadas nos Parques Estaduais da bacia do Ribeira de Iguape e áreas costeiras adjacentes, durante o presente estudo. Mapas foram confeccionados mostrando a localização das Unidades de Conservação visitadas, as espécies amostradas durante este trabalho (Fig. 1) e os registros anteriores de decápodes para a região, depositados no MZUSP (Fig. 2).

\section{Caridea \\ Atyidae}

\section{Atya scabra (Leach, 1815)}

Material. Brasil, São Paulo: bacia do Ribeira de Iguape, município de Cananéia, Parque Estadual da Ilha do Cardoso,

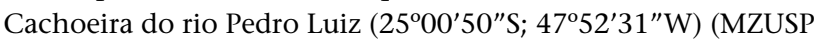
13934) e rio da Cachoeira Grande (MZUSP 15810); bacia da Baixada Santista, município de Peruíbe, Estação Ecológica JuréiaItatins, rio Perequê $\left(24^{\circ} 22^{\prime} 27^{\prime \prime}\right.$; $\left.4^{\circ} 04^{\prime} 03^{\prime \prime} \mathrm{W}\right)$ (MZUSP 13947), Trilha do Fundão $\left(24^{\circ} 23^{\prime} 14^{\prime \prime} \mathrm{S} ; 4^{\circ} 01^{\prime} 02^{\prime \prime} \mathrm{W}\right)$ (MZUSP 13935), rio sem nome (24⒉ $\left.23^{\prime} 09^{\prime \prime} S ; 7^{\circ} 01^{\prime} 07^{\prime \prime} \mathrm{W}\right)$ (MZUSP 13952) e Cacho-

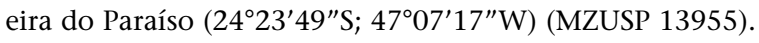

\section{Potimirim glabra (Kingsley, 1878)}

Material. Brasil, São Paulo: bacia da Baixada Santista, município de Peruíbe, Estação Ecológica Juréia-Itatins, rio sem nome (24 $23^{\prime} 09^{\prime \prime}$ S; $\left.47^{\circ} 01^{\prime} 07^{\prime \prime} \mathrm{W}\right)$ (MZUSP 15824) e Trilha do

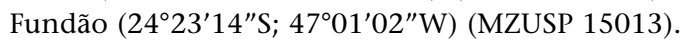

\section{Potimirim potimirim (Müller, 1881)}

Material. Brasil, São Paulo: bacia do Ribeira de Iguape, município de Pariquera-Açu, Parque Estadual Campina do

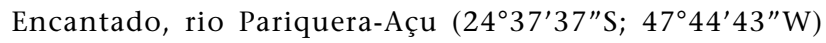

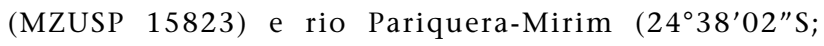
$47^{\circ} 44^{\prime} 14^{\prime \prime} \mathrm{W}$ ) (MZUSP 15827); bacia da Baixada Santista, município de Peruíbe, Estação Ecológica Juréia-Itatins, rio Perequê (24⒉ $\left.22^{\prime} 27^{\prime \prime} S ; 7^{\circ} 04^{\prime} 03^{\prime \prime} \mathrm{W}\right)$ (MZUSP 15014) rio sem nome

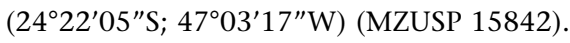

\section{Palaemonidae \\ Macrobrachium acanthurus (Wiegmann, 1836)}

Material. Brasil, São Paulo: bacia do Ribeira de Iguape, município de Pariquera-Açu, Parque Estadual Campina do En-

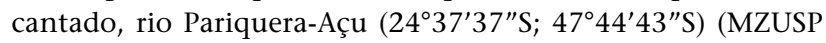
15024) e Pariquera-Mirim (243'02"S; $47^{\circ} 44^{\prime} 14^{\prime \prime}$ W) (MZUSP 15829); bacia do Ribeira de Iguape, município de Ilha Comprida, rio Capivarú $\left(25^{\circ} 00^{\prime} 05^{\prime \prime} S\right.$; 47 $51^{\prime} 53^{\prime \prime} \mathrm{W}$ ) (MZUSP 13941) e

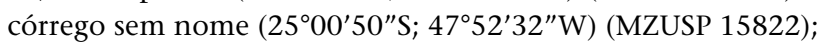



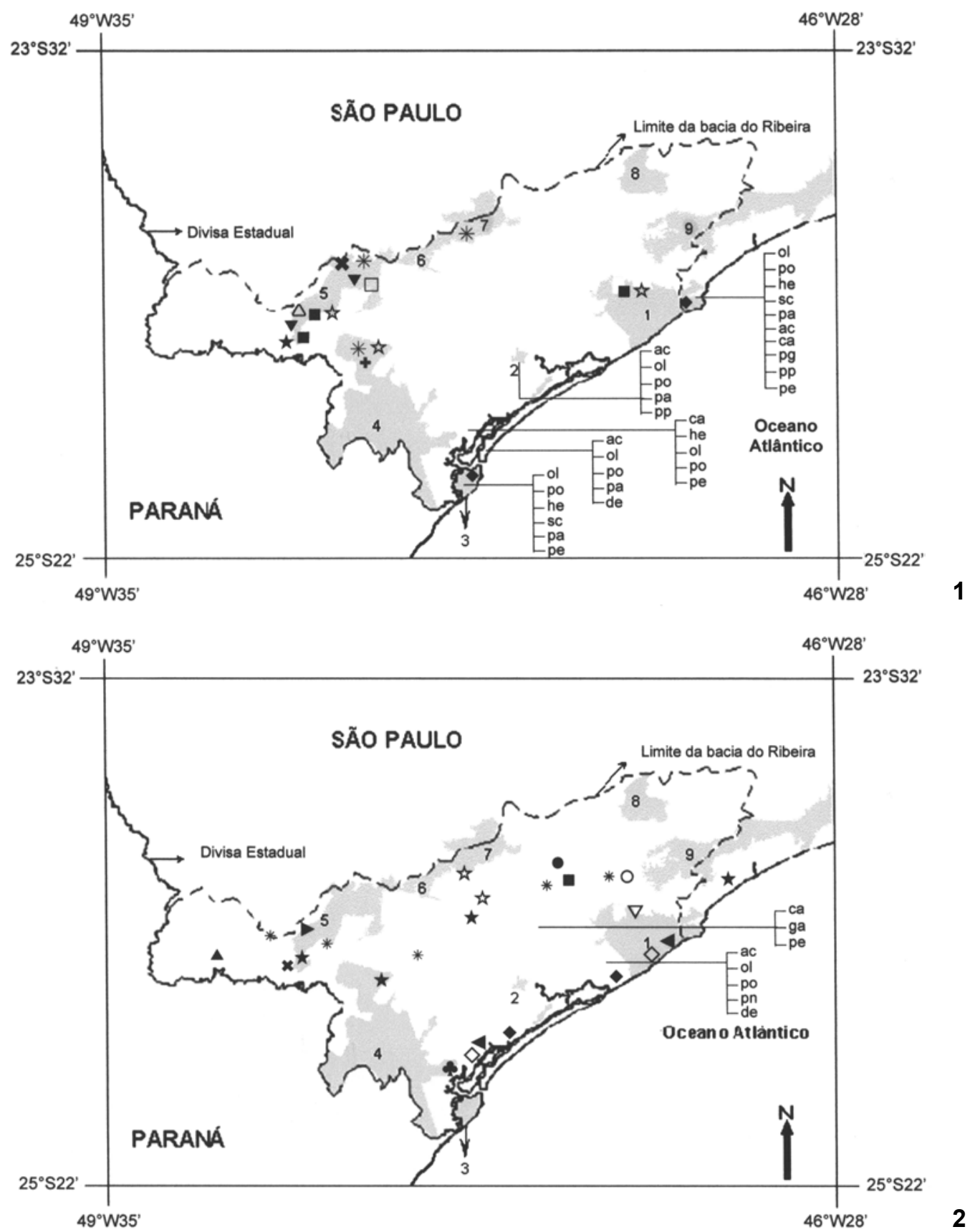

Figuras 1-2. Mapa do sul do estado de São Paulo, com destaque à bacia hidrográfica do Ribeira de Iguape e Unidades de Conservação da região (áreas em cinza), apontando a distribuição das espécies de crustáceos decápodes coletadas neste estudo. Unidades de Conservação: (1) Estação Ecológica Juréia-Itatins, (2) Parque Estadual Campina do Encantado, (3) Parque Estadual da Ilha do Cardoso, (4) Parque Estadual de Jacupiranga, (5) Parque Estadual Turístico do Alto Ribeira (PETAR), (6) Parque Estadual Intervales, (7) Parque Estadual de Carlos Botelho, (8) Parque Estadual de Jurupará, (9) Parque Estadual da Serra do Mar. Espécies: $(\triangle)$ Aegla cavernicola, $(\square)$

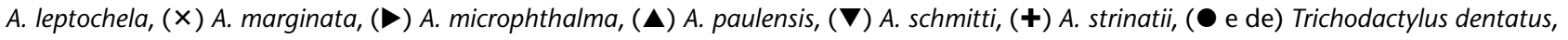
(*) T. fluviatilis, (pn) T. panoplus, ( $\mathbf{\square}$ pe) T. petropolitanus, ( $\bullet$ Pachygrapsus transversus, (ga) Atya gabonensis, (sc) A. scabra, (pp) Potimirim potimirim, (pg) P. glabra, ( $\nabla$ e ac) Macrobrachium acanthurus, ( $)$ M. birai, (ca) M. carcinus, (he) M. heterochirus, (O) M. iheringi, ( $\star$ e ol) M. olfersi, $(\diamond)$ M. petronioi, ( $\star$ e po) M. potiuna, ( $₫$ e pa) Palaemon pandaliformis.

Revista Brasileira de Zoologia 21 (4): 1001-1010, dezembro 2004 
Tabela I. Ocorrência das espécies de crustáceos decápodes coletados na bacia do Ribeira de Iguape e áreas costeiras adjacentes ( $\mathrm{X}=$ encontrado).

\begin{tabular}{|c|c|c|c|c|c|c|c|c|c|}
\hline \multirow[b]{2}{*}{ Táxons } & \multicolumn{9}{|c|}{ Parques estaduais e municípios amostrados } \\
\hline & Juréia-Itatins & $\begin{array}{l}\text { Campina do } \\
\text { Encantado }\end{array}$ & $\begin{array}{l}\text { Carlos } \\
\text { Botelho }\end{array}$ & Intervales & PETAR & Jacupiranga & $\begin{array}{l}\text { Illha do } \\
\text { Cardoso }\end{array}$ & Cananéia & $\begin{array}{c}\text { Ilha } \\
\text { Comprida }\end{array}$ \\
\hline \multicolumn{10}{|l|}{ Aeglidae } \\
\hline Aegla cavernicola & & & & & $x$ & & & & \\
\hline A. leptochela & & & & $x$ & & & & & \\
\hline A. marginata & & & & $x$ & & & & & \\
\hline A. schmitti & & & & $x$ & $x$ & & & & \\
\hline A. strinatii & & & & & & $x$ & & & \\
\hline \multicolumn{10}{|l|}{ Palaemonidae } \\
\hline Macrobrachium acanthurus & $x$ & $x$ & & & & & & & $x$ \\
\hline M. carcinus & $x$ & & & & & & & $x$ & \\
\hline M. heterochirus & $x$ & & & & & & $x$ & $x$ & \\
\hline M. olfersi & $x$ & $x$ & & & & & $x$ & $x$ & $x$ \\
\hline M. potiuna & $x$ & $x$ & & & $\mathrm{x}$ & $x$ & $x$ & $x$ & $x$ \\
\hline Palaemon pandaliformis & $x$ & $x$ & & & & & $x$ & & $x$ \\
\hline \multicolumn{10}{|l|}{ Atyidae } \\
\hline Atya scabra & $x$ & & & & & & $x$ & & \\
\hline Potimirim glabra & $x$ & & & & & & & & \\
\hline P. potimrim & $x$ & $x$ & & & & & & & \\
\hline \multicolumn{10}{|l|}{ Trichodactylidae } \\
\hline Trichodactylus dentatus & & & & & & & & & $x$ \\
\hline T. fluviatilis & & & $x$ & $x$ & & $x$ & & & \\
\hline T. petropolitanus & $x$ & & & & $x$ & & $x$ & $x$ & \\
\hline \multicolumn{10}{|l|}{ Grapsidae } \\
\hline Pachygrapsus transversus & $X$ & & & & & & $X$ & & \\
\hline
\end{tabular}

bacia da Baixada Santista, município de Peruíbe, Estação Ecológica Juréia-Itatins, rio sem nome $\left(24^{\circ} 23^{\prime} 09^{\prime \prime} \mathrm{S} ; 47^{\circ} 01^{\prime} 07^{\prime \prime} \mathrm{W}\right)$ (MZUSP 13942; 15008), rio da Trilha do Fundão (24²3'14"S; $\left.47^{\circ} 01^{\prime} 02^{\prime \prime} \mathrm{W}\right)$ (MZUSP 15845).

\section{Macrobrachium carcinus (Linnaeus 1758)}

Material. Brasil, São Paulo: bacia do Ribeira de Iguape,

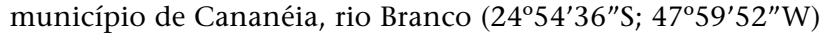
(MZUSP 13930); bacia da Baixada Santista, município de Peruíbe, Estação Ecológica Juréia-Itatins, rio Perequê $\left(24^{\circ} 22^{\prime} 27^{\prime \prime}\right.$;

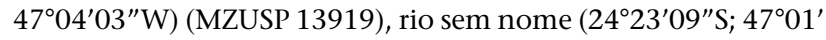
07"W) (MZUSP 15805) e Trilha do Fundão (242' $14^{\prime \prime}$ ' $; 4^{\circ} 01^{\prime}$ 02"W) (MZUSP 13964; 15809).

\section{Macrobrachium heterochirus (Wiegmann, 1836)}

Material. Brasil, São Paulo: bacia do Ribeira de Iguape,

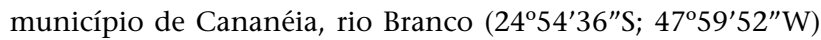
(MZUSP 15022); bacia do Ribeira de Iguape, Parque Estadual da Ilha do Cardoso, cachoeira do rio Pedro Luiz (2500'50"S; $47^{\circ} 52^{\prime} 31^{\prime \prime} \mathrm{W}$ ) (MZUSP 15807) e rio da Cachoeira Grande (MZUSP 15801); bacia da Baixada Santista, município de Peruíbe, Esta- ção Ecológica Juréia Itatins, rio Perequê $\left(24^{\circ} 22^{\prime} 27^{\prime \prime} S ; 7^{\circ} 04^{\prime}\right.$ 03"W) (MZUSP 13953; 15011), Trilha do Fundão (24²3'14"S; $47^{\circ} 01^{\prime} 02^{\prime \prime} \mathrm{W}$ ) (MZUSP 15015), Cachoeira do Paraíso (24²3'49"S; 4707'17”W) (MZUSP 13936; 13959).

\section{Macrobrachium olfersi (Wiegmann, 1836)}

Material. Brasil, São Paulo: bacia do Ribeira de Iguape, município de Pariquera-Açu, Parque Estadual Campina do Encantado, rio Pariquera-Açu ( $24^{\circ} 37^{\prime} 37^{\prime \prime}$; $\left.47^{\circ} 44^{\prime} 43^{\prime \prime} \mathrm{W}\right)$ e rio

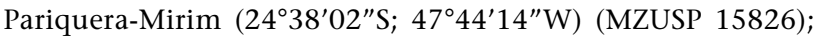
bacia do Ribeira de Iguape, município de Cananéia, rio Branco

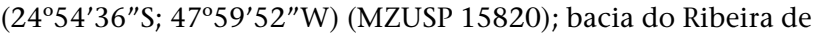
Iguape, município de Ilha Comprida, córrego sem nome

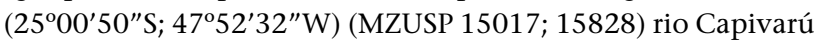

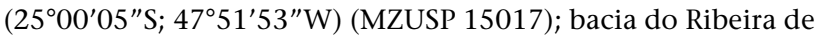
Iguape, Parque Estadual da Ilha do Cardoso, cachoeira do rio Pedro Luiz (2500'50"S; 4752'31"W) (MZUSP 15020), Poço das Antas (MZUSP 15818), rio Perequê (MZUSP 15817) e rio da Cachoeira Grande (MZUSP 15813); bacia da Baixada Santista, município de Peruíbe, Estação Ecológica Juréia-Itatins, rio sem nome (24⒉ $25^{\prime \prime}$; $\left.47^{\circ} 03^{\prime} 17^{\prime \prime} \mathrm{W}\right)$ (MZUSP 15843), rio sem nome

Revista Brasileira de Zoologia 21 (4): 1001-1010, dezembro 2004 


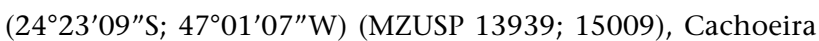
do Paraíso $\left(24^{\circ} 23^{\prime} 49^{\prime \prime}\right.$; $\left.4^{\circ} 7^{\circ} 07^{\prime} 17^{\prime \prime} \mathrm{W}\right)$ (MZUSP 15844), Trilha

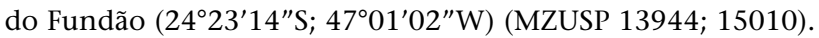

\section{Macrobrachium potiuna (Müller, 1880)}

Material. Brasil, São Paulo: bacia do Ribeira de Iguape, município de Iguape, Estação Ecológica Juréia-Itatins, rio Despraiado (24⒉ $1^{\prime} 55^{\prime \prime}$; $\left.47^{\circ} 18^{\prime} 24^{\prime \prime} \mathrm{W}\right)$ (MZUSP 15012); bacia do Ribeira de Iguape, município de Pariquera-Açu, Parque Estadual Campina do Encantado, rio Pariquera-Açu (24³7'37"S; $47^{\circ} 44^{\prime} 43^{\prime \prime}$ W) (MZUSP 15825) e rio Pariquera-Mirim (243' $02^{\prime \prime}$; $47^{\circ} 44^{\prime} 14^{\prime \prime} \mathrm{W}$ ) (MZUSP 15821); bacia do Ribeira de Iguape, município de Eldorado, Parque Estadual Jacupiranga, rio das

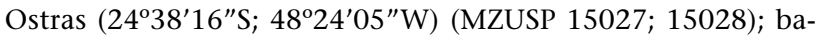
cia do Ribeira de Iguape, município de Iporanga, PETAR, ribeirão de Iporanga (2430'51"S; 48 34'52"W) (MZUSP 15038), caverna Areias I ( $24^{\circ} 35^{\prime} 26^{\prime \prime}$; $48^{\circ} 42^{\prime} 06^{\prime \prime} \mathrm{W}$ ); bacia do Ribeira de Iguape, município de Ilha Comprida, rio sem nome (2500'50"S; 4752'32"W) (MZUSP 15814); bacia do Ribeira de Iguape, município de Cananéia, rio Branco (24²3'14"S; $47^{\circ} 01^{\prime} 02^{\prime \prime} \mathrm{W}$ ) (MZUSP 15819); bacia do Ribeira de Iguape, Parque Estadual da Ilha do Cardoso, rio Santa Cruz (MZUSP 15816), Poço das Antas (MZUSP 15802), rio Perequê (MZUSP 15806) e rio da Cachoeira Grande (MZUSP 15815); bacia da Baixada Santista, município de Peruíbe, Estação Ecológica Juréia-Itatins, rio sem nome $\left(24^{\circ} 22^{\prime} 05^{\prime \prime} S ; 4^{\circ} 03^{\prime} 17^{\prime \prime} \mathrm{W}\right)$ (MZUSP 13522), Ca-

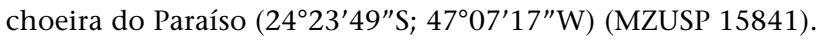

\section{Palaemon pandaliformis (Stimpson, 1871)}

Material. Brasil, São Paulo: bacia do Ribeira de Iguape, município de Cananéia, Parque Estadual da Ilha do Cardoso, rio Pedro Luiz (25 08'24"S; 4800'49"W) (MZUSP 13931; 13932); bacia do Ribeira de Iguape, município de Cananéia, rio Capivarú (2500'05"S; 4751'53”W) (MZUSP 13949); bacia do Ribeira de Iguape, município de Pariquera-Açu, Parque Estadual Campina do Encantado, rio Pariquera-Açu (2437'37"S; $47^{\circ} 44^{\prime} 43^{\prime \prime}$ W) (MZUSP 13954; 15800); bacia da Baixada Santista, município de Peruíbe, Estação Ecológica Juréia-Itatins, rio sem

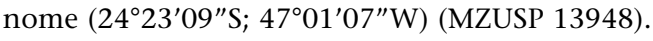

\section{Anomura$$
\text { Aeglidae }
$$

\section{Aegla cavernicola Türkay, 1972}

Material. Brasil, São Paulo: bacia do Ribeira de Iguape, município de Iporanga, PETAR, caverna Areias II (26 $01^{\prime} 04^{\prime \prime}$; 484'ㄱ"W) (MZUSP 15034).

\section{Aegla leptochela Bond-Buckup \& Buckup, 1994}

Material. Brasil, São Paulo: bacia do Ribeira de Iguape, município de Iporanga, Parque Estadual Intervales, caverna dos Paiva (2416'36"S; 48 26'31"W) (MZUSP 15033).

\section{Aegla marginata Bond-Buckup \& Buckup, 1994}

Material. Brasil, São Paulo: bacia do Ribeira de Iguape, município de Guapiara, Parque Estadual Intervales, rio Bocaina

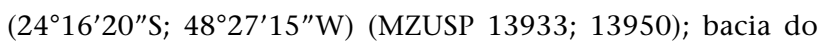
Ribeira de Iguape, município de Iporanga, Parque Estadual Intervales, córrego Água Comprida ( $\left.24^{\circ} 17^{\prime} 35^{\prime \prime} \mathrm{S} ; 48^{\circ} 25^{\prime} 05^{\prime \prime} \mathrm{W}\right)$ (MZUSP 15035), caverna dos Paiva $\left(24^{\circ} 16^{\prime} 36^{\prime \prime} \mathrm{S} ; 48^{\circ} 26^{\prime} 31^{\prime \prime} \mathrm{W}\right)$ e Gruta Colorida (MZUSP 15032).

\section{Aegla schmitti Hobbs III, 1979}

Material. Brasil, São Paulo: bacia do Ribeira de Iguape, município de Iporanga, PETAR, rio Betari / Roncador (24 $31^{\prime}$ 58"S; 4841'58”W) (MZUSP 13625; 13927; 13943; 15036), caverna Santana $\left(24^{\circ} 31^{\prime} 58^{\prime \prime} S\right.$; 48 41'58”W) (MZUSP 13570; $13572 ; 13623)$, saída da caverna Areias II $\left(26^{\circ} 01^{\prime} 04^{\prime \prime}\right.$; 48 47'27"W) (MZUSP 15812); bacia do Ribeira de Iguape, município de Iporanga, Parque Estadual Intervales, rio do Carmo / Lajeado (2418'25"S; 48²4'52"W) (MZUSP 13569; 13574; $13575 ; 13624 ; 13626 ; 13628 ; 13938 ; 15029)$.

\section{Aegla strinatii Türkay, 1972}

Material. Brasil, São Paulo: bacia do Ribeira de Iguape, município de Eldorado, Parque Estadual Jacupiranga, rio das

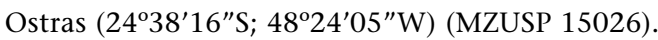

\section{Brachyura \\ Grapsidae}

\section{Pachygrapsus transversus (Gibbes, 1850)}

Material. Brasil, São Paulo: bacia do Ribeira de Iguape, município de Cananéia, Parque Estadual da Ilha do Cardoso, rio Pedro Luiz (25 08'24"S; 48 00'49"W) (MZUSP 15023); bacia da Baixada Santista, município de Peruíbe, rio sem nome

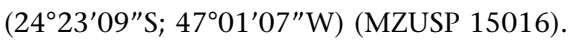

\section{Trichodactylidae}

\section{Trichodactylus dentatus H. Milne Edwards, 1853}

Material. Brasil, São Paulo: bacia do Ribeira de Iguape, município de Ilha Comprida, córrego sem nome (2500'22"S; $47^{\circ}$ 52'25"W) (MZUSP 15018).

\section{Trichodactylus fluviatilis Latreille, 1828}

Material. Brasil, São Paulo: bacia do Ribeira de Iguape, município de Iporanga, Parque Estadual Intervales, rio do

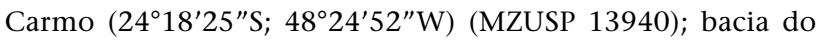
Ribeira de Iguape, município de Eldorado, Parque Estadual Jacupiranga, rio das Ostras / caverna do Diabo (243' $16^{\prime \prime}$ 'S; $48^{\circ} 24^{\prime} 05^{\prime \prime W}$ ) (MZUSP 15025); bacia do Ribeira de Iguape, município de Sete Barras, Parque Estadual Carlos Botelho, rio Preto $\left(24^{\circ} 10^{\prime} 18^{\prime \prime}\right.$; $\left.47^{\circ} 57^{\prime} 42^{\prime \prime} \mathrm{W}\right)$ (MZUSP 15846) e rio Água Para-

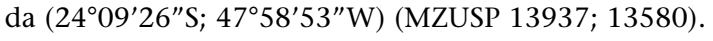

\section{Trichodactylus petropolitanus (Göldi, 1886)}

Material. Brasil, São Paulo: bacia do Ribeira de Iguape, município de Iguape, Estação Ecológica Juréia-Itatins, rio Despraiado ( $24^{\circ} 21^{\prime} 55^{\prime \prime} \mathrm{S} ; 4^{\circ} 18^{\prime} 24^{\prime \prime} \mathrm{W}$ ) (MZUSP 15021); bacia do Ribeira de Iguape, município de Iporanga, PETAR, ribeirão de Iporanga (2430'51"S; 48³4'52"W) (MZUSP 13945; 15037), 
saída da caverna Areias II (2601'04"S; 48²7'27"W) (MZUSP 15798); bacia do Ribeira de Iguape, município de Cananéia,

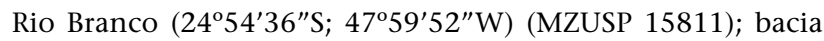
do Ribeira de Iguape, município de Cananéia, Parque Estadual da Ilha do Cardoso, rio Santa Cruz (MZUSP 15799), Poço das Antas (MZUSP 15803), rio Perequê (MZUSP 15808) e rio da Cachoeira Grande (MZUSP 15804); bacia da Baixada Santista, município de Peruíbe, Estação Ecológica Juréia-Itatins, rio sem

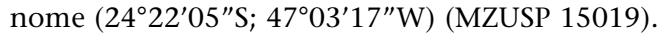

\section{DISCUSSÃO}

Segundo MagalHães (1999b), são reconhecidas 12 espécies de camarões Macrobrachium para o estado de São Paulo. No presente trabalho representantes desse gênero foram encontrados nos Parques Estaduais Campina do Encantado, PETAR, Jacupiranga, Ilha do Cardoso, além da Estação Ecológica JuréiaItatins e municípios de Ilha Comprida e Cananéia, totalizando cinco espécies (Tab. I). A ocorrência dessas espécies foi consideravelmente ampliada, especialmente na região da Estação Ecológica Juréia-Itatins, nos Parques Estaduais da Ilha do Cardoso, Campina do Encantado e nos municípios de Cananéia e Ilha Comprida (Fig. 1).

Com exceção de um lote de M. acanthurus (MZUSP 9560), proveniente da Serra da Juréia, o registro das espécies $M$. carcinus, M. heterochirus, M. potiuna e M. olfersi representa um incremento significativo no número de espécies na região da E. E. Juréia-Itatins. Nos Parques Estaduais Campina do Encantado, Ilha do Cardoso e municípios de Cananéia e Ilha Comprida, ainda não havia sido realizado um levantamento sistemático de espécies de camarões Macrobrachium, fazendo com que os resultados aqui apresentados sejam os primeiros registros dessas espécies.

No MZUSP há outros lotes provenientes do Vale do Ribeira de Iguape, que embora se localizem fora da área investigada neste trabalho, complementam o mapa de distribuição desses camarões na região. São eles: Macrobrachium acanthurus do município de Iguape (MZUSP 222; 569) e rio Ribeira (MZUSP 7484); M. olfersi do rio das Ostras (MZUSP 13586), foz do rio Saibadela (MZUSP 13564; 13565), município de Iguape (MZUSP 726), rio Iporanga, município de Iporanga (MZUSP 13590) e município de Peruíbe (MZUSP 1368); Macrobrachium potiuna do rio Ipiranga, município de Sete Barras (MZUSP 13561; 13556); Rio Quilombo (MZUSP 9944, 13557, 13559), rio Ribeira de Iguape, município de Iguape (MZUSP 7482), rio Saibadela (MZUSP 13558); Macrobrachium carcinus do município de Registro (MZUSP 6188) (Fig. 2).

Duas outras espécies de camarões palemonídeos não foram encontradas durante o presente estudo: Macrobrachium birai Lobão, Melo \& Fernandes, 1986 (MZUSP 8027, 9561) e Macrobrachium petronioi Melo, Lobão \& Fernandes, 1986 (MZUSP 8034, 8035, 8037, 8038), redescritas por Melo et al. (1988) cuja localidade tipo é o Rio Branco, no município de Cananéia e que também ocorrem no rio Verde, na Estação Eco- lógica Juréia-Itatins (Lobão et al. 1992, Lombardi et al. 1996) (Fig. 2). Segundo Melo et al. (1988), M. petronioi é muito semelhante a M. potiuna, o mesmo ocorrendo entre M. birai e M. olfersi. O rio Branco foi alvo de duas amostragens durante o presente trabalho e os indivíduos capturados foram identificados como M. olfersi ou M. potiuna.

Macrobrachium iheringi não foi encontrado nas Unidades de Conservação estudadas, mas sua ocorrência na região foi verificada por exemplares provenientes de Miracatu, presentes na coleção do MZUSP (MZUSP 7483) (Fig. 2).

Macrobrachium nattereri (Heller, 1862) foi assinalado para o estado de São Paulo por Luederwaldt (1919a,b). Um lote dessa espécie foi encontrado na coleção do MZUSP (MZUSP 1465) e, possivelmente, integraria o material que o citado autor examinou para registrar essa ocorrência. Entretanto, HoLthuIs (1952) recomendou que as identificações de Luederwaldt necessitavam de confirmação, pois espécimes do Museu Paulista (hoje MZUSP), anteriormente identificados como M. nattereri, na realidade tratavam-se de M. potiuna. Após examinarmos o lote $\mathrm{n}^{\circ} 1465$ e, com base na morfologia do segundo par de quelípodos e na fórmula rostral, constatamos que os espécimes que compõem o referido lote são de fato M. potiuna.

A riqueza de Macrobrachium spp. diminuiu à medida que os pontos de coleta tornavam-se mais distantes do litoral (Fig. 1), o que tem relação com a estratégia reprodutiva dos dois grandes grupos desse gênero. No primeiro grupo, aqui representado por M. acanthurus, M. olfersi, M. heterochirus e M. carcinus, há uma dependência da água salobra para completar o desenvolvimento larval, restringindo a distribuição à região costeira. No outro grupo, representado por M. potiuna e M. iheringi, o desenvolvimento larval transcorre totalmente em água doce, possibilitando a colonização de águas continentais, o que explica a menor diversidade de camarões do gênero Macrobrachium em regiões mais afastadas da faixa litorânea (BUENo \& RoDRIGUEs 1995).

No Museu de Zoologia da Universidade de São Paulo existem lotes de M. olfersi provenientes dos municípios de Iporanga (MZUSP 13549) e Eldorado (MUSP 13568), os quais localizamse a dezenas de quilômetros do mar. GnASPINI \& Trajano (1994) também relataram a presença desta espécie na caverna Alambari de Cima (SP-11). Tais populações podem ter se estabelecido nessas localidades mediante migração de indivíduos adultos, mas é provável que suas larvas jamais contribuirão para o processo de recrutamento, devido a distância dos estuários onde completariam seu desenvolvimento com sucesso.

No Vale do Ribeira, a espécie Palaemon pandaliformis foi encontrada em rios da Estação Ecológica Juréia-Itatins, Parque Estadual Campina do Encantado, Parque Estadual da Ilha do Cardoso e município de Ilha Comprida (Tab. I). Essa espécie foi freqüentemente encontrada muito próxima a estuários, com exceção do rio Pariquera-Açu, no Parque Estadual Campina do Encantado, situado na planície costeira, a $60 \mathrm{Km}$ do litoral (Fig. 1).

O desenvolvimento larval de uma população de $P$. pandaliformis da Venezuela foi obtido tanto em água salgada 
(GAMBA, 1992) como em água doce (GAMBA, 1998). Aparentemente, a salinidade da água não é um fator limitante para as larvas de $P$. pandaliformis que eclodem no P.E. Campina do Encantado, não afetando o processo de recrutamento de indivíduos na população. Dados da coleção do MZUSP registram a presença da espécie em Cananéia (MZUSP 6488) (Fig. 2).

Com relação aos atiídeos, o presente relato da ocorrência de Atya scabra no Vale do Ribeira é inédito, já que em registros anteriores esta espécie havia sido relatada apenas para a região de São Sebastião, no litoral norte do estado (GALVão \& Bueno 2000).

Os exemplares de $A$. scabra coletados no Vale do Ribeira foram encontrados próximos às regiões litorâneas do Parque Estadual da Ilha do Cardoso e Estação Ecológica Juréia-Itatins (Fig. 1), habitando riachos de água corrente e limpa com fundo de pedras utilizadas como abrigo. Tal distribuição litorânea ocorre pela dependência que as larvas têm da água salobra para completarem seu desenvolvimento (Hobbs \& Hart 1982, AвRUnHosa \& Moura 1988, Galvão \& Bueno 2000). É pertinente mencionar que a coleção do Museu de Zoologia da USP contém, ainda, um lote de Atya gabonensis Giebel, 1875 coletado no município de Registro (MZUSP 11443), fora dos limites das áreas de conservação estudadas neste trabalho (Fig. 2).

Os espécimes do gênero Potimirim Holthuis, 1954 habitam as margens dos rios, sempre associados à vegetação marginal ou ao folhiço acumulado em seu leito. Foram coletadas e identificadas duas espécies, Potimirim glabra e $P$. potimirim, em rios dos Parques Estaduais Campina do Encantado e Estação Ecológica Juréia-Itatins (Tab. I). Apesar de muito comuns na região, os resultados aqui apresentados constituem o primeiro registro destas espécies no Vale do Ribeira de Iguape e áreas costeiras adjacentes.

Atualmente, as espécies do gênero Potimirim apresentam questões de natureza taxonômica ainda não elucidadas. Em 1959, Villalobos apontou similaridades entre $P$. glabra e $P$. brasiliana Villalobos, 1959, levando Smalley (1963) a propor a sinonímia entre tais espécies, justificando que Holthuis (1954) e Villalobos (1959) não haviam examinado machos de P. glabra. Esta sugestão de sinonímia foi aceita por CHACE \& Hobbs (1969), Rodriguez (1980) e Holthuis (1986), sendo que este último recomenda estudos adicionais para uma melhor avaliação. MANNING \& Hobbs Jr. (1977) е Villalobos (1982), por outro lado, consideraram $P$. glabra e $P$. brasiliana como espécies distintas. Outros autores também destacaram a grande semelhança entre $P$. mexicana (Saussure, 1857) e $P$. potimirim, sugerindo a sinonímia destas espécies (Abele 1972, Gore et al. 1978, Villalobos 1982).

No presente estudo os exemplares identificados como $P$. glabra, seguem a proposta de Smalley (1963), С насе \& Hobbs (1969) e Rodriguez (1980), que consideram P. brasiliana sinônimo júnior de $P$. glabra. Os exemplares identificados como $P$. potimirim, apesar de semelhantes morfologicamente a $P$. mexicana, não resultaram em dúvidas durante a identificação, já que a distribuição geográfica de $P$. mexicana não abrange o Brasil e a morfologia dos espécimes coletados é idêntica àquela descrita para P. potimirim por MÜLler (1892b) e VILLALOBos (1959).

Com relação aos caranguejos tricodactilídeos, MAGALHãES (1999b) compilou todas as espécies registradas para o estado de São Paulo. Quatro dessas espécies [T. fluviatilis, T. petropolitanus, T. dentatus e T. panoplus (von Martens, 1869)] podem ser encontradas na bacia do rio Ribeira de Iguape. O registro da ocorrência de T. panoplus em São Paulo está baseada em um único lote (MZUSP 313) com três machos e uma fêmea, cuja procedência seria a cidade de Iguape (Luederwaldt, 1919a,b) (Fig. 2). Entretanto, no presente estudo essa espécie não foi coletada em nenhuma das sete áreas de proteção estudadas, reforçando as suspeitas sobre a validade dessa ocorrência.

O presente trabalho ampliou sobremaneira a distribuição de Trichodactylidae no Vale do Ribeira, já que não havia nenhum registro de caranguejos dulcícolas em rios da Estação Ecológica Juréia-Itatins, Parques Estaduais Carlos Botelho e Ilha do Cardoso, além dos municípios de Cananéia e Ilha Comprida (Tab. I, Fig. 1).

A maioria das espécies de caranguejos tricodactilídeos brasileiros habita rios de planície em altitudes inferiores a trezentos metros (Magalhães, 1999a). Contudo, no Vale do Ribeira foram registrados exemplares de Trichodactylus fluviatilis e $T$. petropolitanus em rios localizados na Serra de Paranapiacaba, em altitudes superiores a quinhentos metros. O desenvolvimento direto desses crustáceos, constatado pela primeira vez por MüLLER (1892a), representa um importante fator na conquista de águas continentais, cabeceiras dos rios e rios de planície costeira.

Outras áreas não investigadas neste trabalho, mas com registro de caranguejos tricodactilídeos na coleção do Museu de Zoologia são: T. fluviatilis provenientes da cidade de Apiaí (MZUSP 6328), Eldorado (MZUSP 2281), Iporanga (MZUSP 1753, 1766, 1768, 7354, 9509), Juquiá (MZUSP 1750) e Miracatu (MZUSP 10742); T. petropolitanus de Juquiá (MZUSP 1785, 6345, 6346, 6367) e Registro (MZUSP 9701); T. dentatus de Iguape (MZUSP 454) e Juquiá (MZUSP 9499) (Fig. 2).

O gênero Pachygrapsus Randall, 1840 é representado por três espécies no Brasil (Melo, 1996). Até o momento, a única espécie registrada no Vale do Ribeira é Pachygrapsus transversus, cuja distribuição abrange todo o litoral do Brasil, habitando principalmente a zona do entre marés de maguezais e praias arenosas, e na água acumulada em bromeliáceas (Melo 1996, Masunari \& Dubiaski-Silva 1998). Furtado-Ogawa (1977) verificaram uma maior captura de exemplares no médio-litoral, adotando como intertidal a distribuição dessa espécie. No presente trabalho, a ocorrência de $P$. transversus nos rios costeiros de água doce da Estação Ecológica Juréia-Itatins e Parque Estadual da Ilha do Cardoso (Tab. I, Fig. 1) pode ser considerada temporária ou acidental em função do reduzido número de exemplares amostrados $(\mathrm{n}=3)$. P. transversus já havia sido coletada, no Vale do Ribeira, nos municípios de Cananéia (MZUSP 6149) e Iguape (MZUSP 483) (Fig. 2). No entanto, este trabalho amplia o conhecimento da área de ocorrência dessa espécie, já que ela não havia sido ainda registrada na Estação Ecológica

Revista Brasileira de Zoologia 21 (4): 1001-1010, dezembro 2004 
Juréia-Itatins e Parque Estadual da Ilha do Cardoso.

No caso dos eglídeos, existem dez espécies registradas para o estado de São Paulo (Bond-Buckup \& Buckup 1994, MAgALHÃEs 1999b), dentre as quais sete são registradas para o Vale do Ribeira, sendo algumas espécies comuns em cavernas da região (Trajano \& Gnaspini-Neto 1990). Dessas sete espécies, cinco foram coletadas em rios dos Parques Estaduais Intervales, PETAR e Jacupiranga (Tab. I, Fig. 1). A espécie A. microphthalma Bond-Buckup \& Buckup, 1994 não foi coletada por ser uma espécie troglóbia, habitando o interior da caverna Santana, de difícil acesso. Contudo, há lotes desta espécie no Museu de Zoologia (MZUSP 7405, 7408), coletados no passado (Fig. 2). Da mesma forma, A. paulensis Schmitt, 1942 não foi encontrada em nenhuma das Unidades de Conservação investigadas, embora exista um lote desta espécie (MZUSP 11061) proveniente do município de Ribeira, não investigado no presente trabalho (Fig. 2).

Aegla schmitti foi a espécie de maior abundância e distribuição no Vale do Ribeira, sendo encontrada em grande número em rios do PETAR e Intervales, além das seguintes cavernas: Santana, Temimina II (MZUSP 7321, 9771), Gruta da Aegla (MZUSP 9776) e Água Suja (MZUSP 7323, 7411). Aegla marginata foi coletada apenas em rios e cavernas do Parque Estadual Intervales, embora Bond-BUCKUP \& BUCKUP (1994) mencionem a existência de material proveniente da Casa de Pedra, no PETAR (MZUSP 7409, 9779, 9780, 9781, 9783) (Fig. 2). Por sua vez, $A$. strinatii é endêmica do rio das Ostras, formador da Caverna do Diabo, localizada no Parque Estadual de Jacupiranga (BondBuckup \& Buckup 1994) (Fig. 1).

As demais espécies capturadas (A. cavernicola e A. leptochela), têm distribuição restrita às suas localidades-tipo: Caverna Areais I e II e Gruta dos Paiva, respectivamente (Fig. 1) (BondBuckup \& Buckup 1994). Essas duas espécies, juntamente com A. microphthalma, encontrada na Caverna Santana, no PETAR (MZUSP 7312, 7405, 7408) (Fig. 2), constituem as espécies troglóbias da porção paulista da Província Espeológica do Vale do Ribeira. Seus indivíduos possuem modificações fisiológicas (crescimento lento, grande longevidade, baixa fertilidade) e morfológicas (redução dos olhos, alongamento dos apêndices, despigmentação) que não os permite viver no meio externo tornando-os muito vulneráveis a perturbações ambientais (PINTO-DA-Rocha 1993, Trajano \& GNaSPINI 2001).

\section{AGRADECIMENTOS}

Os autores gostariam de expressar seus sinceros agradecimentos à Fábio Kiyohara e Emerson C. Mossolin pela ajuda na coleta dos espécimes e agradável companhia durante as viagens de campo; à Profa. Dra. Georgina Bond-Buckup, Prof. Dr. Célio Magalhães e Isabel C. Arantes, que confirmaram a identificação dos exemplares; e ao Prof. Dr. Gustavo Augusto S. de Melo, que gentilmente nos permitiu consultar os lotes depositados no Museu de Zoologia da Universidade de São
Paulo. À FAPESP (Fundação de Amparo à Pesquisa do Estado de São Paulo), pela concessão de auxílio financeiro (Programa BIOTA-FAPESP, processo $\mathrm{n}^{\circ}$ 98/05073-4) e bolsa de Mestrado (processo nº 00/02119-5); à Coordenação do Programa de PósGraduação em Zoologia e Departamento de Zoologia do IB / USP, pelo apoio logístico. Finalmente, muito obrigado a todos os funcionários dos Parques Estaduais visitados, bem como do Instituto Florestal, que sempre nos receberam cordialmente.

\section{REFERÊNCIAS BIBLIOGRÁFICAS}

Aвele, L.G. 1972. Introduction of two fresh-water Decapod Crustaceans (Hymenosomatidae and Atyidae) into Central and North America. Crustaceana, Leiden, 23: 209-213.

Abrunhosa, F.A. \& M.G. Moura. 1988. O Completo Desenvolvimento Larval do Camarão Atya scabra (Leach) (Crustacea: Decapoda: Atyidae), Cultivado em Laboratório. Arquivos de Ciências do Mar, Fortaleza, 27: 127-146.

Bond-Buckup, G. \& L. Buckup. 1994. A Família Aeglidae (Crustacea, Decapoda, Anomura). Arquivos de Zoologia do Museu de Zoologia da Universidade de São Paulo, São Paulo, 32 (4): 159-346.

Bueno, S.L.S. \& S.A. Rodrigues. 1995. Abbreviated larval development of the freshwater praw, Macrobrachium iheringi (Ortman, 1897) (Decapoda, Palaemonidae), reared in the laboratory. Crustaceana, Leiden, 68 (6): 665-686.

Chace JR., F.A. \& H.H. Hobbs. 1969. The freshwater and Terrestrial Decapod Crustaceans of the West Indies with Special Reference to Dominica. United States National Museum Bulletin, Washington, 292: 1-256.

Elmor, M.R.D.; V.L. Lobão \& W.C. Valenti, 1981. Consumo de oxigênio por Macrobrachium acanthurus (Wiegmann, 1836) como subsídio ao seu transporte e cultivo. Boletim do Instituto de Pesca, São Paulo, 8: 65-78.

Furtado-Ogawa, E. 1977. Notas Biológicas sobre Pachygrapsus transversus (Gibbes, 1850) no Estado do Ceará (Crustacea: Brachyura). Arquivos de Ciências do Mar, Fortaleza, 17 (2): 107-113.

GALVÃo, R. \& S.L.S. Bueno. 2000. Population structure and reproductive biology of the Camacuto shrimp, Atya scabra (Leach, 1815) (Decapoda, Caridea, Atyidae), from São Sebastião, Brazil. Crustacean Issues, Leiden, 12: 291-299.

GambA, A. 1992. Desarrollo larval de Palaemon pandaliformis (Decapoda: Palaemonidae) em dos salinidades. Memorias del VII Simposio Latinoamericano y II Encuentro Venezuelano de Acuicultura, Barquisimeto, p. 152-159. . 1998. The Larval Development of a Fresh-water Prawn, Palaemon pandaliformis (Stimpson, 1871), under Laboratory Conditions (Decapoda, Palaemonidae). Crustaceana, Leiden, 71 (1): 9-35.

Genofre, G.S. \& V.L. LobÃo. 1978. Macrobrachium holthuisi, sp. n., a new species of shrimp (Decapoda, Macrura). Crustaceana, Leiden, 35 (3): 273-276. 
Gnaspini, P. \& E. Trajano. 1994. Brazilian Cave Invertebrates, with a Checklist of Troglomorphic Taxa. Revista Brasileira de Entomologia, São Paulo, 38 (3/4): 549-584.

Gore, R.H.; G.R. Kulczycki \& P.A. Hastinhg. 1978. A Second Occurrence of the Brazilian Freshwater shrimp Potimirim potimirim, along the Central Eastern Florida Coast. Florida Scientist, Florida, 41 (1): 57-61.

Новвs, H.H. \& C.W. Hart. 1982. The Shrimp genus Atya (Decapoda, Atyidae). Smithsonian Contribution to Zoology, Washington, 364: 1-143.

Holthuis, L.B. 1952. A general revision of the Palaemonidae (Crustacea, Decapoda, Natantia) of the Americas. II. The subfamily Palaemoninae. Occasional Paper of the Allan Hancock Foundation, Los Angeles, 12: 1-396.

- 1954. On a Collection of Decapod Crustacea from the Republic of El Salvador (Central America). Zoologische Verhandelingen, Leiden, 23: 1-43.

- 1986. Fresh-water shrimps of the Family Atyidae from Western Colombia. Journal of Crustacean Biology, Lawrence, 6 (3): 438-445.

IHERING, H. von. 1897. Os camarões de água doce do Brasil. Revista do Museu Paulista, São Paulo, 2: 421-432.

Kensley, B. \& I. Walker. 1982. Palaemonid Shrimps from the Amazon Basin, Brazil (Crustacea: Decapoda: Natantia). Smithsonian Contributions to Zoology, Washington, 362: $1-28$.

Lobão, V.L.; J.V. Lombardi; S.G. Melo; H.P. Barros; E. HortênCio \& E.A. Roverso. 1992. Estudos Populacionais de Macrobrachium birai Lobão, Melo \& Fernandes e Macrobrachium petronioi Melo, Lobão \& Fernandes das Regiões de Cananéia e Juréia (SP-Brasil) I. Dinâmica da Reprodução. Boletim do Instituto de Pesca, São Paulo, 19: 49-62.

Lombardi, J.V.; V.L. Lobão \& E.A. Roverso. 1996. Estudos Populacionais de Macrobrachium birai Lobão, Melo \& Fernandes e Macrobrachium petronioi Melo, Lobão \& Fernandes das Regiões de Cananéia e Juréia (SP-Brasil) II. Dinâmica do Crescimento. Boletim do Instituto de Pesca, São Paulo, 23: 47-54.

Luederwaldt, H. 1919a. Os Manguesaes de Santos. Revista do Museu Paulista, São Paulo, 11: 309-409.

1919b. Lista dos crustáceos superiores (Thoracostraca) do Museu Paulista que foram encontrados no Estado de São Paulo. Revista do Museu Paulista, São Paulo, 11: 427-453.

Magalhães, C. \& M. TÜrkAY 1996. Taxonomy of the Neotropical freshwater crab family Trichodactylidae I. The generic system with description of some new genera (Crustacea: Decapoda: Brachyura). Senckenbergiana biologica, Frankfurt, 75 (1/ 2): 63-95.

Magalhães, C. 1999a. Família Trichodactylidae (caranguejos braquiúros da água doce), p. 486-490. In: L. Buckup \& G. BondBuckup (Ed.). Os Crustáceos do Rio Grande do Sul. Porto Alegre, UFRGS, VI+496p.
- 1999b. Crustáceos Decápodes, p. 127-133. In: D. Ismael; W.C. Valenti; T. Matsumura-Tundisi; O. Rocha (Eds). Biodiversidade do Estado de São Paulo, Brasil. Invertebrados de Água Doce. São Paulo, FAPESP, vol. 4, XXII+ $176 \mathrm{p}$.

Manning R.B. \& H.H. HobBs JR. 1977. Decapoda, p. 157-162. In: S.H. Hubert (Ed.). Biota Aquatica de Sudamerica Austral. San Diego, San Diego State University, XIV+342p.

Masunari, S. \& J. Dubiaski-Silva. 1998. Crustacea Decapoda da Praia Rochosa da Ilha do Farol, Matinhos, Paraná. II. Distribuição Espacial de Densidade das Populações. Revista Brasileira de Zoologia, Curitiba, 15 (3): 643-664.

MCNamara, J.C. 1981. Morphological organization of crustacean pigmentary effectors. Biological Bulletin, New York, 161: 270-280.

McNamara, J.C. \& A. Sesso. 1982. Pigment biogenesis in freshwater shrimp ventral nerve chord chromatophores. Cell Tissue Research, Berlin, 222: 167-175.

Melo, G.A.S. 1996. Manual de Identificação dos Brachyura (caranguejos e siris) do Litoral Brasileiro. São Paulo, FAPESP, 604p.

. 2003. Famílias Atyidae, Palaemonidae e Sergestidae, p. 289-409. In: G.A.S. Melo (Ed.). Manual de Identificação dos Crustacea Decapoda de água doce do Brasil. São Paulo, Editora Loyola, 429p.

Melo, G.A.S.; V.L. LobÃo \& W.M. Fernandes. 1988. Redescrição de Macrobrachium birai, Lobão, Melo \& Fernandes e de Macrobrachium petronioi, Melo, Lobão \& Fernandes (Crustacea, Decapoda) Palemonídeos da região sul do estado de São Paulo; Brasil. Boletim do Instituto de Pesca, São Paulo, 15 (1): 89-97.

Melo, S.G. \& A.L. Brossi-Garcia. 1999. Postembryonic development of Macrobrachium petronioi (Caridea, Palaemonidae) in the laboratory. Journal of Crustacean Biology, Lawrence, 19 (3): 622-642.

Moreira, G.S.; J.C. McNamara; P.S. Moreira \& M. Weinrich. 1980. Temperature and salinity effects on the respiratory metabolism of the first zoeal stage of Macrobrachium holthuisi Genofre \& Lobão (Decapoda: Palaemonidae). Journal of Experimental Marine Biology and Ecology, Amsterdam, 47: 141-148.

Mossolin, E.C. \& S.L.S. BuEno. 2002. Reproductive Biology of Macrobrachium olfersi (Decapoda, Palaemonidae) in São Sebastião, Brazil. Journal of Crustacean Biology, Lawrence, 22 (2): 367-376.

Mossolin, E.C. \& S.L.S. Bueno. 2003. Relative Growth of the second pereiopod in Macrobrachium olfersi (Wiegmann, 1836) (Decapoda, Palaemonidae). Crustaceana, Leiden, 76 (3): 363-376.

Müller, F. 1880. Palaemon Potiuna. Ein Beispiel abgekürzter Verwandlung. Zoologischer Anzeiger, Leipzip, 3: 152-157.

- 1892a. Trichodactylus, siri de água doce sem metamorfose. Archivos do Museu Nacional do Rio de Janeiro, 
Rio de Janeiro, 8: 125-133.

, 1892b. O Camarão miúdo do Itajahy Ayoida potimirim. Archivos do Museu Nacional do Rio de Janeiro, Rio de Janeiro, 8: 155-178.

Oliveira, L.P.H. 1945. Verificação da existência de Atya scabra Leach, camarão d'água doce da família Atyidae, Crustacea, no Nordeste do Brasil. Memórias do Instituto Oswaldo Cruz, Rio de Janeiro, 43 (1): 177-190.

Ortmann, A. 1897. Os camarões de água doce da América do Sul. Revista do Museu Paulista, São Paulo, 2: 173-216.

Ostrovski, M.C.; K.M.L. Fonseca; T.C.G. Silva-Ferreira \& F.J.P. Matos. 1996. Macrobrachium denticulatum sp. nov. a new species of shrimp from the São Francisco Basin, Northeastern Brazil. Crustaceana, Leiden, 69 (3): 359-367.

PINTO-DA-Rocha, R. 1993. Invertebrados Cavernícolas da Porção Meridional da Província Espeológica do Vale do Ribeira, Sul do Brasil. Revista Brasileira de Zoologia, Curitiba, 10 (2): 229-255.

Rocha, S.S. \& S.L.S. Bueno. 2000. Probopyrus floridensis Richardson, 1904 (Isopoda, Epicaridea) parasitizing the Freshwater Prawn Macrobrachium potiuna (Müller, 1880), from São Paulo, Brazil. Naupilus, Botucatu, 8 (1): 133-140.

Rodriguez, G. 1980. Los Crustaceos Decapodos de Venezuela. Caracas, Instituto Nacional de Invertigaciones Científicas, 494p.

SAWAYA, M.P. 1946. Sobre alguns camarões d'água doce do Bra- sil. Boletim da Faculdade de Filosofia, Ciências e Letras da Universidade de São Paulo, Zoologia, São Paulo, 11: 393-407.

Schmitr, W.L. 1942. The Species of Aegla, endemic South American Fresh-water Crustacean. Proceedings of the United States National Museum, Washington, 91 (31-32): 431-524.

Smalley A.E. 1963. The genus Potimirim in Central America (Crustacea, Atyidae). Revista de Biologia Tropical, Costa Rica, 11 (2): 177-183.

Trajano, E. \& P. Gnaspini-Netto. 1990. Composição da Fauna Cavernícola Brasileira, com uma Análise da Distribuição dos Táxons. Revista Brasileira de Zoologia, São Paulo, 7 (3): 383-407.

. 2001. Cavernas, p. 189-200. In: C. Leonel (Ed.). Intervales. São Paulo, Fundação para a Conservação e Produção Florestal do Estado de São Paulo, 240p.

Villalobos, A. 1959. Contribuicion al conocimiento de los Atyidae de Mexico II (Crustacea, Decapoda). Estudio de algunas especies del genero Potimirim (= Ortmania), con descripción de una especie nueva en Brasil. Annales del Instituto de Biologia de la Universidad de Mexico, Cidade do México, 30: 269-330.

- 1982. Decapoda, p. 215-239. In: S.H. Hulbert \& A. Villalobos-Figueroa (Eds). Aquatic Biota of Mexico, Central America and the West Indies. San Diego, San Diego State University, 529p.

Recebido em 26.II.2004; aceito em 22.XI.2004.

Revista Brasileira de Zoologia 21 (4): 1001-1010, dezembro 2004 